\title{
Percutaneous Mitral Valve Intervention Using MitraClip for Functional Mitral Regurgitation and Heart Failure
}

\author{
Hidehiro Kaneko, ${ }^{1,2}$ MD, Hiroyuki Kiriyama, ${ }^{1}$ MD, Tatsuya Kamon, ${ }^{1}$ MD, Hidetaka Itoh, ${ }^{1}$ MD, \\ Satoshi Kodera, ${ }^{1}$ MD, Katsuhito Fujiu, ${ }^{1,2}$ MD, Masao Daimon, ${ }^{1,3}$ MD, Hiroyuki Morita, ${ }^{1}$ MD, \\ Masaru Hatano, ${ }^{1,4}$ MD and Issei Komuro, ${ }^{1}$ MD
}

\begin{abstract}
Summary
Functional mitral regurgitation (FMR) frequently coexists with left ventricular systolic dysfunction and advanced heart failure, and typically has poor clinical outcomes. Although various therapeutic options including cardiac resynchronization therapy and surgical mitral intervention, have been proposed, an optimal treatment strategy for functional mitral regurgitation has not yet been established. Over the last decade, transcatheter mitral valve repair using MitraClip has emerged as a novel alternative therapeutic option for functional mitral regurgitation. In 2018, the COAPT trial demonstrated that MitraClip treatment reduced rehospitalization due to heart failure and all-cause death in patients with functional mitral regurgitation and heart failure. As a consequence, the MitraClip has become a very promising potential treatment for functional mitral regurgitation. In this review, we discuss and summarize the current status and future perspectives of the treatment for functional mitral regurgitation and heart failure.
\end{abstract}

Key words: Mitral regurgitation, Structural heart disease intervention, COAPT trial

(Int Heart J 2021; 62: 4-8)

$\mathrm{F}$ unctional mitral regurgitation (FMR) currently is a widely discussed and researched topic in cardiovascular medicine. Based on pathological features, $\overline{\mathrm{MR}}$ is categorized into degenerative and functional MR. Degenerative (primary) MR occurs secondary to lesions of the mitral valve (MV) leaflets, chordae tendineae and papillary muscles, whereas functional (secondary) MR occurs in the absence of organic MV disease. Left ventricular (LV) dilatation (remodeling) primarily caused by ischemic or dilated cardiomyopathy leads to apical and posterior displacement of the papillary muscles, MV leaflet tethering and annular dilatation and causes FMR in a structurally normal (or near-normal) MV.

Owing to the underlying mechanism, FMR is commonly observed in patients with LV systolic dysfunction (LVSD). Moreover, FMR causes further deterioration of LV function and results in unfavorable clinical outcomes caused by a progressive spiral of LV remodeling.

In association with underlying LV remodeling, FMR commonly coexists with heart failure (HF). Koelling, et $a l{ }^{1)}$ reported that patients with FMR represent a high-risk subset of patients with LV dysfunction. Trichon, et al. reported similar results ${ }^{2)}$ in that significant FMR was commonly observed in patients with LV dysfunction and HF, and FMR was independently associated with lower survival rates. FMR is more commonly observed in patients with advanced HF. For example, moderate-to-severe FMR occurs in approximately $33 \%$ of patients with advanced $\mathrm{HF}$ in whom cardiac resynchronization therapy (CRT) is indicated. ${ }^{3,4)}$ Previous studies report that severe FMR occurred in $25 \%$ of patients with ischemic and nonischemic dilated cardiomyopathy and that a close association was observed between severe FMR and long-term clinical outcomes in patients with both ischemic and dilated cardiomyopathy. ${ }^{5)}$ Moreover, our previous studies have shown that in Japanese patients, FMR was commonly observed in patients with HF and LV dysfunction, resulting in unfavorable clinical outcomes. ${ }^{6,7)} \mathrm{We}$ previously reported that significant FMR (moderate-to-severe) was associated with higher incidence rates of all-cause death (hazard ratio 2.2), cardiovascular death (hazard ratio 2.4), and hospital admissions for HF (hazard ratio 1.8). Additionally, significant FMR was observed in approximately $17 \%$ of patients with $\mathrm{LV}$ dysfunction (LV ejection fraction $[\mathrm{EF}] \leq 40 \%$ ) and was associated with a higher incidence of the composite end-point including all-cause death and/or hospital admission for HF (hazard ratio 1.6). These results indicate that FMR is an important and challenging issue in cardio-

From the 'Department of Cardiovascular Medicine, Graduate School of Medicine, The University of Tokyo, Tokyo, Japan, ${ }^{2}$ Department of Advanced Cardiology, Graduate School of Medicine, The University of Tokyo, Tokyo, Japan, ${ }^{3}$ Department of Clinical Laboratory, The University of Tokyo Hospital, Tokyo, Japan and ${ }^{4}$ Department of Therapeutic Strategy for Heart Failure, Graduate School of Medicine, The University of Tokyo, Tokyo, Japan.

Address for correspondence: Hidehiro Kaneko, MD, Department of Cardiovascular Medicine, The University of Tokyo Hospital, 7-3-1 Hongo, Bunkyo-ku, Tokyo, 113-8655, Japan. E-mail: kanekohidehiro@gmail.com

Received for publication October 27, 2020. Revised and accepted October 27, 2020.

doi: $10.1536 /$ ihj.20-712

All rights reserved by the International Heart Journal Association. 
vascular clinical practice in Japan (particularly, for $\mathrm{HF}$ and LV dysfunction).

\section{Therapeutic Strategy for Functional Mitral Regurgitation and Heart Failure}

To date, the optimal medical treatment for patients with FMR and LVSD is unknown. The cornerstone of treatment for FMR is treatment of underlying LV dysfunction and HF, including guideline-directed medical therapy (GDMT) for HF, as well as CRT.

GDMT for HF (in patients with reduced EF) is the first-line treatment for FMR. Beta-blockers and reninangiotensin system inhibitors should be prescribed for all patients with FMR and LV dysfunction. Several studies have reported on the efficacy of beta-blockers in patients with FMR. ${ }^{8,9)}$ A small randomized trial has reported that carvedilol treatment decreased the severity of FMR in patients with dilated cardiomyopathy. ${ }^{10)}$ Levine, et al. showed that uptitration of renin-angiotensin system inhibitors could improve severe FMR in patients with dilated cardiomyopathy in association with reverse remodeling. ${ }^{11)}$ However, the morbidity and mortality rates in patients with FMR remain high despite GDMT. ${ }^{12}$

CRT also shows a positive effect in patients with FMR. CRT reduces FMR in patients with HF and left bundle branch block. ${ }^{13)}$ Bommel, et al. reported a reduction in FMR in patients categorized as high-risk surgical patients undergoing CRT and that the improved FMR was associated with better survival. ${ }^{14)}$ The Multicenter InSync Randomized Clinical Evaluation trial showed that CRT resulted in sustained reduction in FMR in patients with severe $\mathrm{HF}$ with reduced $\mathrm{EF}$ and a wide $\mathrm{QRS}$ complex. ${ }^{15)}$ However, the improvement in severe FMR was observed in only approximately $50 \%$ of patients undergoing CRT. ${ }^{14,16)}$

Surgical MV repair is the established standard treatment for degenerative MR and is a class I recommendation for degenerative MR. ${ }^{17,18)}$ However, the effects of MV surgery including MV repair and replacement on FMR remain uncertain. MV surgery is effective for acute reduction of FMR, and Bach, et al. reported that MV surgery improved LVEF and cardiac output with symptomatic improvement of $\mathrm{HF}$ in patients with dilated cardiomyopathy. ${ }^{19)}$ However, several studies have shown that MV surgery did not improve survival rates in patients with FMR. $\mathrm{Wu}$, et al. reported no clear mortality benefit conferred by MV annuloplasty for significant MR with severe LV dysfunction. ${ }^{20)}$ Compared with MV repair, chordal-sparing MV replacement results in sustained improvement in MR; however, no significant difference was observed between these procedures with respect to LV reverse remodeling or survival rates. ${ }^{21)}$ Smith, et al. reported that the addition of MV repair to coronary artery bypass graft surgery did not improve survival rates in patients with moderate ischemic MR. ${ }^{22)}$ Therefore, per the latest guidelines, the recommendation level for MV surgery in patients with FMR is not high (class IIb). ${ }^{17,18)}$

\section{MitraClip for Functional Mitral Regurgitation and Heart Failure}

Over the last decade, transcatheter mitral valve repair (TMVR) has emerged as a novel alternative in patients with severe MR who are considered high-risk or prohibitively high-risk patients for conventional MV surgery. The MitraClip device (Abbott Vascular, Santa Clara, CA, USA) is the most commonly used among the various devices available for TMVR. MitraClip is a polyestercovered cobalt-chromium clip inserted via the femoral vein. This technique mimics the Alfieri stitch ("doubleorifice" repair). MitraClip is inserted into the left atrium via the trans-septal route, and this device grasps the free edges of the anterior and posterior MV leaflets. Grasping both MV leaflets using MitraClip creates a "doubleorifice" and serves as a useful minimally invasive technique to reduce MR.

The Endovascular Valve Edge-to-Edge Repair Study II (EVEREST II) trial included 278 patients with grades 3+/4+ MR who were randomized into the MitraClip group or MV surgery group. The MitraClip technique was significantly safer than MV surgery, and the New York Heart Association functional class and overall survival rates were similar between the aforementioned groups over a 4year follow-up. ${ }^{23)}$ Subsequent prospective studies such as the ACCESS-Europe-A Two-Phase Observational Study of the MitraClip System in Europe (ACCESS-EU) registry and the transcatheter mitral valve interventions (TRAMI) registry reported acceptable outcomes with respect to $\mathrm{HF}$ symptoms; however, to date, the beneficial effects of MitraClip on long-term outcomes remain unproven. Moreover, these registries included patients with both degenerative MR and FMR; therefore, the efficacy of the MitraClip device for FMR remains debatable.

Notably, approximately $70 \%$ of the patients investigated in the real-world registries including the ACCESSEU and the TRAMI registry showed FMR etiology. ${ }^{24,25)}$ FMR commonly occurs concomitantly with advanced HF and LV remodeling ${ }^{6,7)}$ therefore, MitraClip is considered a useful therapeutic option even in patients with HF. Nevertheless, the clinical benefit of MitraClip in treating FMR remains unclear; therefore, based on the latest European Guidelines, the recommendation level for MitraClip for FMR remains low (recommendation class IIb, evidence level $\mathrm{C}^{18)}$ ). Among the patients included in the EVEREST II trial, only $27 \%$ showed FMR. Therefore, when the MitraClip was approved by the United States Food and Drug Administration (FDA) in 2013, the indication for its use was limited to patients with degenerative MR with a prohibitively high risk for MV surgery. From this perspective, clinical evidence supporting MitraClip in patients with FMR and HF is strongly warranted.

In 2018, 2 randomized trials investigating the efficacy of MitraClip for FMR presented pivotal results. The Percutaneous Repair with the MitraClip Device for Severe Functional/Secondary Mitral Regurgitation (MITRA-FR) study, which included 304 patients and compared GDMT with GDMT plus MitraClip for FMR and HF, reported no difference in the primary composite endpoint including rehospitalization for $\mathrm{HF}$ and all-cause death after 1 year. ${ }^{26}$ 
In contrast, the Cardiovascular Outcomes Assessment of the MitraClip Percutaneous Therapy for Heart Failure Patients with Functional Mitral Regurgitation (COAPT) trial, which included 614 patients and compared GDMT with GDMT plus MitraClip for FMR and HF, reported a significant reduction in rehospitalization for $\mathrm{HF}$ (hazard ratio 0.53 ) and all-cause death (hazard ratio 0.62) in the MitraClip group after 2 years. ${ }^{27)}$ Despite the similar study design, the results of the MITRA-FR and COAPT trials are completely different.

The diametrically opposite results between the MITRA-FR and COAPT trials could be attributed to the following features: (1) The degree of baseline MR and the LV volume differed between patients included in the MITRA-FR and COAPT trials. The severity of MR at baseline levels was greater in patients enrolled in the COAPT trial than in patients enrolled in the MITRA-FR trial (mean effective regurgitant orifice area $41 \pm 15 \mathrm{~mm}^{2}$ versus $31 \pm 10 \mathrm{~mm}^{2}$ ). Additionally, the degree of LV dilatation was lesser in patients enrolled in the COAPT trial than in patients enrolled in the MITRA-FR trial $(101 \pm 34$ $\mathrm{mL} / \mathrm{m}^{2}$ versus $135 \pm 35 \mathrm{~mL} / \mathrm{m}^{2}$ ). These findings suggest a significantly more severe degree of MR but a smaller LV volume in patients enrolled in the COAPT trial than in patients enrolled in the MITRA-FR. Grayburn, et al. reported that the COAPT trial primarily included patients with "disproportionate" MR and that these patients benefitted from MitraClip, whereas the MITRA-FR trial primarily included patients with "proportionate MR" who did not benefit from MitraClip. ${ }^{28)}$ (2) Efficacy of MitraClip in MR reduction was superior in the COAPT trial. Postprocedural MR grade $\geq 3+$ after MitraClip occurred more commonly in the MITRA-FR than in the COAPT trial $(9 \%$ versus $5 \%)$. Additionally, $\mathrm{MR}$ grade $\geq 3+$ a year after MitraClip was also more common in the MITRA-FR than in the COAPT trial (17\% versus 5\%). Differences in MR reduction could contribute to the differences in clinical outcomes observed between the trials. (3) Procedural complications occurred more commonly in the MITRA-FR than in the COAPT trial (15\% versus $9 \%)$. Thus, we need to carefully interpret the different results of the COAPT trial and the MITRA-FR trial. However, it can be concluded that MitraClip can serve as an effective treatment for a "specific subset" of patients with FMR and HF, and the optimal patient selection criteria is the next issue to be discussed. The Randomized Study of the MitraClip Device in Heart Failure Patients With Clinically Significant Functional Mitral Regurgitation (RESHAPE-HF2) trial (NCT02444338) is currently underway in Europe to assess a similar proposition (the efficacy of MitraClip for FMR). Hopefully, the results of this RESHAPE-HF2 trial (which are expected in the near future) will provide a better understanding of this issue. Moreover, the Multicenter, Randomized, Controlled Study to Assess Mitral vAlve reconsTrucTion for advancEd Insufficiency of Functional or iscHemic ORigiN trial (NCT02371512), which compares clinical outcomes between patients with MR undergoing MV surgery and MitraClip, is currently underway. This study is also of clinical interest.

\section{Future Perspectives in Japan}

Since April 2018, MitraClip has been commercially available as a novel therapeutic option for MR in Japan. The etiology that warrants MitraClip use in Japanese patients is FMR and HF (similar to the indications for patients in Europe). Nevertheless, MitraClip is contraindicated in Japan in patients with LVEF $<30 \%$ based on the inclusion criteria outlined in a Pharmaceuticals and Medical Devices Agency-approved trial. ${ }^{29)}$ In contrast, LVEF values do not serve as deciding indications/contraindications for MitraClip in Europe and the United States. Epidemiological data indicate that MV surgery was contraindicated in approximately $50 \%$ of patients with severe symptomatic MR, which was primarily attributed to impaired LV function. ${ }^{30)}$ Moreover, Bach, et al. reported that only $16 \%$ of patients with FMR underwent MV surgery. ${ }^{31)}$ Therefore, an unmet medical need exists in the management of patients with FMR with impaired LV function, and the MitraClip device is expected to address this unmet medical need. Moreover, based on the results of the COAPT trial, the FDA approved MitraClip use for FMR and HF in March 2019. Therefore, MitraClip is expected to gain wider acceptance to treat FMR and HF not only in Europe but also in the United States. From this perspective, the Japanese guideline excluding patients with LVEF $<30 \%$ is arguable. This unique contraindication in Japan apparently stems from the fact that baseline LV dysfunction is associated with poor outcomes after MV surgery, ${ }^{32)}$ and MV surgery is not strongly recommended (class IIb recommendation) for severe degenerative MR in patients with LVEF $\leq 30 \%{ }^{33)}$ However, previous studies have shown that MitraClip clinically benefits even patients with reduced $\mathrm{LVEF}^{34,35)}$ The COAPT trial also showed that MitraClip improved clinical outcomes in patients with FMR and $\mathrm{HF}$ with $\mathrm{LVEF}<30 \%$. $^{27)}$ Furthermore, the efficacy of the MitraClip device was confirmed even in CRT nonresponders (mean LVEF 19\%) ${ }^{36)}$ and patients with end-stage HF (mean LVEF 27\%). ${ }^{37)}$ With regard to the current status of management of patients with advanced HF in Japan, the waiting period for heart transplantation remains long, and to date, implantation of a ventricular assist device as destination therapy is not approved. Therefore, practical treatment options remain limited for patients with advanced HF in Japan. In this current scenario, MitraClip is a novel therapeutic option not only to treat severe MR but also in patients with advanced HF with FMR. However, data from 2 German studies has shown that approximately $50 \%$ of patients with FMR and HF who underwent MitraClip in Germany would be ineligible to undergo $\mathrm{Mi}$ traClip in Japan owing to low LVEF. ${ }^{38)}$ Therefore, it is necessary to reconsider the indications/contraindications for MitraClip in patients with reduced LVEF in Japan. Notably, previous studies have reported several cardiac and non-cardiac prognostic factors (other than LVEF) in patients undergoing MitraClip. ${ }^{39-46)}$ Thus, patient selection criteria for MitraClip should be carefully evaluated based on the clinical characteristics of each patient. In addition to the MitraClip device, numerous emerging devices for TMVR or MV replacement are available. ${ }^{47)}$ Further investigation is warranted not only with respect to MitraClip 
but also for these novel emerging devices. Several questions remain unanswered including the optimal timing of intervention (early versus too late), the effect of MV intervention in patients with end-stage $\mathrm{HF}$, and the optimal medical management before and after MV intervention. Despite several challenges that need to be overcome, efforts must be continued for the advancement of medical treatment for patients with FMR and HF.

\section{Disclosure}

Conflicts of interest: None.

\section{References}

1. Koelling TM, Aaronson KD, Cody RJ, Bach DS, Armstrong WF. Prognostic significance of mitral regurgitation and tricuspid regurgitation in patients with left ventricular systolic dysfunction. Am Heart J 2002; 144: 524-9.

2. Trichon BH, Felker GM, Shaw LK, Cabell CH, O'Connor CM. Relation of frequency and severity of mitral regurgitation to survival among patients with left ventricular systolic dysfunction and heart failure. Am J Cardiol 2003; 91: 538-43.

3. Dickstein K, Vardas PE, Auricchio A, et al. 2010 Focused Update of ESC Guidelines on device therapy in heart failure: an update of the 2008 ESC Guidelines for the diagnosis and treatment of acute and chronic heart failure and the 2007 ESC guidelines for cardiac and resynchronization therapy. Developed with the special contribution of the Heart Failure Association and the European Heart Rhythm Association. Eur Heart J 2010; 31: 2677-87.

4. Di Biase L, Auricchio A, Mohanty P, et al. Impact of cardiac resynchronization therapy on the severity of mitral regurgitation. Europace 2011; 13: 829-38.

5. Rossi A, Dini FL, Faggiano P, et al. Independent prognostic value of functional mitral regurgitation in patients with heart failure. A quantitative analysis of 1256 patients with ischaemic and non-ischaemic dilated cardiomyopathy. Heart 2011; 97: 1675-80.

6. Kaneko H, Suzuki S, Uejima T, et al. Prevalence and the longterm prognosis of functional mitral regurgitation in Japanese patients with symptomatic heart failure. Heart Vessels 2014; 29: 801-7.

7. Kaneko H, Suzuki S, Uejima T, et al. Functional mitral regurgitation and left ventricular systolic dysfunction in the recent era of cardiovascular clinical practice, an observational cohort study. Hypertens Res 2014; 37: 1082-7.

8. Capomolla S, Febo O, Gnemmi M, et al. Beta-blockade therapy in chronic heart failure: diastolic function and mitral regurgitation improvement by carvedilol. Am Heart J 2000; 139: 596608.

9. Comin-Colet J, Sanchez-Corral MA, Manito N, et al. Effect of carvedilol therapy on functional mitral regurgitation, ventricular remodeling, and contractility in patients with heart failure due to left ventricular systolic dysfunction. Transplant Proc 2002; 34: 177-8.

10. Lowes BD, Gill EA, Abraham WT, et al. Effects of carvedilo on left ventricular mass, chamber geometry, and mitral regurgitation in chronic heart failure. Am J Cardiol 1999; 83: 1201-5.

11. Levine AB, Muller C, Levine TB. Effects of high-dose lisinopril-isosorbide dinitrate on severe mitral regurgitation and heart failure remodeling. Am J Cardiol 1998; 82: 1299-301.

12. Agricola E, Ielasi A, Oppizzi M, et al. Long-term prognosis of medically treated patients with functional mitral regurgitation and left ventricular dysfunction. Eur J Heart Fail 2009; 11: 5817.
13. Breithardt OA, Sinha AM, Schwammenthal E, et al. Acute effects of cardiac resynchronization therapy on functional mitral regurgitation in advanced systolic heart failure. J Am Coll Cardiol 2003; 41: 765-70.

14. van Bommel RJ, Marsan NA, Delgado V, et al. Cardiac resynchronization therapy as a therapeutic option in patients with moderate-severe functional mitral regurgitation and high operative risk. Circulation 2011; 124: 912-9.

15. St John Sutton MG, Plappert T, Abraham WT, et al. Effect of cardiac resynchronization therapy on left ventricular size and function in chronic heart failure. Circulation 2003; 107: 198590.

16. Onishi T, Onishi T, Marek JJ, et al. Mechanistic features associated with improvement in mitral regurgitation after cardiac resynchronization therapy and their relation to long-term patient outcome. Circ Heart Fail 2013; 6: 685-93.

17. Nishimura RA, Otto CM, Bonow RO, et al. 2017 AHA/ACC Focused Update of the 2014 AHA/ACC Guideline for the Management of Patients With Valvular Heart Disease: A Report of the American College of Cardiology/American Heart Association Task Force on Clinical Practice Guidelines. Circulation 2017; 135: e1159-95.

18. Baumgartner H, Falk V, Bax JJ, et al. 2017 ESC/EACTS Guidelines for the management of valvular heart disease. Eur Heart $\mathrm{J}$ 2017; 38: 2739-91.

19. Bach DS, Bolling SF. Early improvement in congestive heart failure after correction of secondary mitral regurgitation in endstage cardiomyopathy. Am Heart J 1995; 129: 1165-70.

20. Wu AH, Aaronson KD, Bolling SF, Pagani FD, Welch K, Koelling TM. Impact of mitral valve annuloplasty on mortality risk in patients with mitral regurgitation and left ventricular systolic dysfunction. J Am Coll Cardiol 2005; 45: 381-7.

21. Goldstein D, Moskowitz AJ, Gelijns AC, et al. Two-Year Outcomes of Surgical Treatment of Severe Ischemic Mitral Regurgitation. N Engl J Med 2016; 374: 344-53.

22. Smith PK, Puskas JD, Ascheim DD, et al. Surgical treatment of moderate ischemic mitral regurgitation. N Engl J Med 2014; 371: 2178-88.

23. Mauri L, Foster E, Glower DD, et al. 4-year results of a randomized controlled trial of percutaneous repair versus surgery for mitral regurgitation. J Am Coll Cardiol 2013; 62: 317-28.

24. Maisano F, Franzen O, Baldus S, et al. Percutaneous mitral valve interventions in the real world: early and 1-year results from the ACCESS-EU, a prospective, multicenter, nonrandomized post-approval study of the MitraClip therapy in Europe. J Am Coll Cardiol 2013; 62: 1052-61.

25. Eggebrecht H, Schelle S, Puls M, et al. Risk and outcomes of complications during and after MitraClip implantation: Experience in 828 patients from the German TRAnscatheter mitral valve interventions (TRAMI) registry. Catheter Cardiovasc Interv 2015; 86: 728-35.

26. Obadia JF, Messika-Zeitoun D, Leurent G, et al. Percutaneous Repair or Medical Treatment for Secondary Mitral Regurgitation. N Engl J Med 2018; 379: 2297-306.

27. Stone GW, Lindenfeld J, Abraham WT, et al. Transcatheter Mitral-Valve Repair in Patients with Heart Failure. N Engl J Med 2018; 379: 2307-18.

28. Grayburn PA, Sannino A, Packer M. Proportionate and Disproportionate Functional Mitral Regurgitation: A New Conceptual Framework That Reconciles the Results of the MITRA-FR and COAPT Trials. JACC Cardiovasc Imaging 2019; 12: 353-62.

29. Hayashida K, Yasuda S, Matsumoto T, et al. AVJ-514 TrialBaseline Characteristics and 30-Day Outcomes Following MitraClip((R)) Treatment in a Japanese Cohort. Circ J 2017; 81: 1116-22.

30. Mirabel M, Iung B, Baron G, et al. What are the characteristics of patients with severe, symptomatic, mitral regurgitation who are denied surgery? Eur Heart J 2007; 28: 1358-65.

31. Bach DS, Awais M, Gurm HS, Kohnstamm S. Failure of guideline adherence for intervention in patients with severe mitral re- 
gurgitation. J Am Coll Cardiol 2009; 54: 860-5.

32. Enriquez-Sarano M, Tajik AJ, Schaff HV, Orszulak TA, Bailey KR, Frye RL. Echocardiographic prediction of survival after surgical correction of organic mitral regurgitation. Circulation 1994; 90: 830-7.

33. Nishimura RA, Otto CM, Bonow RO, et al. 2014 AHA/ACC guideline for the management of patients with valvular heart disease: a report of the American College of Cardiology/American Heart Association Task Force on Practice Guidelines. J Am Coll Cardiol 2014; 63: e57-185.

34. Kaneko H, Neuss M, Schau T, Weissenborn J, Butter C. Impact of left ventricular systolic dysfunction on the outcomes of percutaneous edge-to-edge mitral valve repair using MitraClip. Heart Vessels 2016; 31: 1988-96.

35. Lesevic H, Sonne C, Braun D, et al. Acute and Midterm Outcome After MitraClip Therapy in Patients With Severe Mitral Regurgitation and Left Ventricular Dysfunction. Am J Cardiol 2015; 116: 749-56.

36. Auricchio A, Schillinger W, Meyer S, et al. Correction of mitral regurgitation in nonresponders to cardiac resynchronization therapy by MitraClip improves symptoms and promotes reverse remodeling. J Am Coll Cardiol 2011; 58: 2183-9.

37. Franzen O, van der Heyden J, Baldus S, et al. MitraClip(R) therapy in patients with end-stage systolic heart failure. Eur J Heart Fail 2011; 13: 569-76.

38. Kaneko H, Kitamura M, Neuss M, et al. MitraClip in Patients With Mitral Regurgitation and Left Ventricular Ejection Fraction $<30 \%$ - Potential Implications for the Treatment of Patients in Japan. Circ J 2018; 82: 2672-5.

39. Neuss M, Schau T, Schoepp M, et al. Patient selection criteria and midterm clinical outcome for MitraClip therapy in patients with severe mitral regurgitation and severe congestive heart failure. Eur J Heart Fail 2013; 15: 786-95.
40. Ohno Y, Attizzani GF, Capodanno D, et al. Association of tricuspid regurgitation with clinical and echocardiographic outcomes after percutaneous mitral valve repair with the MitraClip System: 30-day and 12-month follow-up from the GRASP Registry. Eur Heart J Cardiovasc Imaging 2014; 15: 1246-55.

41. Matsumoto T, Nakamura M, Yeow WL, et al. Impact of pulmonary hypertension on outcomes in patients with functional mitral regurgitation undergoing percutaneous edge-to-edge repair. Am J Cardiol 2014; 114: 1735-9.

42. Kaneko H, Neuss M, Weissenborn J, Butter C. Role of Right Ventricular Dysfunction and Diabetes Mellitus in N-terminal pro-B-type Natriuretic Peptide Response of Patients With Severe Mitral Regurgitation and Heart Failure After MitraClip. Int Heart J 2017; 58: 225-31.

43. Kaneko H, Neuss M, Weissenborn J, Butter C. Impact of residual mitral regurgitation after MitraClip implantation. Int J Cardiol 2017; 227: 813-9.

44. Neuss M, Schau T, Isotani A, Pilz M, Schopp M, Butter C. Elevated Mitral Valve Pressure Gradient After MitraClip Implantation Deteriorates Long-Term Outcome in Patients With Severe Mitral Regurgitation and Severe Heart Failure. JACC Cardiovasc Interv 2017; 10: 931-9.

45. Kaneko H, Neuss M, Schau T, Weissenborn J, Butter C. Interaction between renal function and percutaneous edge-to-edge mitral valve repair using MitraClip. J Cardiol 2017; 69: 476-82.

46. Kaneko H, Neuss M, Okamoto M, Weissenborn J, Butter C. Impact of Preprocedural Anemia on Outcomes of Patients With Mitral Regurgitation Who Underwent MitraClip Implantation. Am J Cardiol 2018; 122: 859-65.

47. Taramasso M, Kuwata S, Rodriguez Cetina Biefer H, Nietlispach F, Maisano F. Percutaneous mitral valve repair and replacement: complementary or competitive techniques? EuroIntervention 2016; 12: Y97-101. 